\title{
Eclipsing binary distances to the edge of the Local Group
}

\author{
Alceste Z. Bonanos \\ Institute of Astronomy, Astrophysics, Space Applications and Remote Sensing, \\ National Observatory of Athens, Metaxa \& Pavlou, Palaia Penteli 15236, Greece \\ email: bonanos@astro.noa.gr
}

\begin{abstract}
Eclipsing binaries are powerful tools for determining the fundamental parameters of stars and, therefore, for measuring accurate, independent distances to nearby galaxies. I present distance measurements that are in progress based on early-type eclipsing binary systems in several Local Group galaxies at a range of metallicities, and discuss the strengths of the method, the limitations and possible sources of systematic error. The goal is to establish several local stepping stones along the extragalactic distance ladder, which will help calibrate the Cepheid period-luminosity relation and thus resolve the ongoing controversy about the distance scale, with its ramifications for cosmology and stellar ages.
\end{abstract}

Keywords. distance scale, stars: distances, binaries: eclipsing, stars: fundamental parameters, Local Group

\section{Eclipsing Binaries as Independent Distance Indicators}

Eclipsing binaries (EBs) have the potential to establish fundamental distances to the edge of the Local Group with an accuracy of 5\% for single systems (Paczyński 1997) and even better if many binaries are observed. EBs offer much information about their physical parameters (see the reviews by Andersen 1991; Torres et al. 2010). We can measure the sizes of the stars from the velocities and the eclipse durations, the luminosities from the radii and surface temperatures, and the distance to the system from the magnitudes. This requires good modeling of the binary system. In particular, if the two stars are nearly identical, the uncertainties in determining the orbital and stellar surface parameters become minor. Thus, we can derive a distance which is primarily limited by the accuracy with which we can measure the light curve and the radial velocities. Such a distance is completely independent of the usual distance ladder.

The strengths of the EB method for measuring distances, compared to other methods, are that it provides a distance that is direct, geometric, and independent of the distance scale. The method relies on model atmospheres for measuring the stellar metallicity and the flux, which are fitted using state-of-the-art non-LTE (local thermodynamic equilibrium) model atmospheres in the case of early-type systems. Another important strength of the method is that it yields a direct determination of the reddening and reddening law along the line of sight to the system, which is impossible to independently estimate using most other methods. Finally, the EB method, in principle, also allows for direct determination of blending, which is estimated from the light curve, by fitting for the "third light.' Blending is a significant source of systematic error affecting many other distance indicators (e.g., Cepheids; Chavez et al. 2012).

Measuring distances based on EBs to the edge of the Local Group requires identifying bright, eclipsing, double-lined spectroscopic binaries of OB type in the target galaxies. Such early-type stars have absolute magnitudes in the range $-7<M_{V}<-5$ mag, which 
correspond to apparent magnitudes of $V \in[18-20]$ mag. At these magnitudes, the highresolution spectroscopy $(R>3000)$ and signal-to-noise ratio $>30$ required to measure accurate radial velocities, are achievable with 6-10 m-class telescopes out to $1 \mathrm{Mpc}$. Note that such early-type systems are usually found in short-period systems $(<10$ days), which facilitates photometric observations. Multi-band time-series photometry is needed to model the light curves and multi-band out-of-eclipse absolute photometry, including at near-infrared wavelengths, is needed to establish the flux of the system and to estimate the reddening and reddening law. Finally, state-of-the-art non-LTE model atmospheres, (e.g., FASTWIND) are required to model the component spectra, since surface brightness calibrations are not yet available for OB-type stars.

Table 1. Census of extragalactic eclipsing binaries.

\begin{tabular}{lccc}
\hline Galaxy & Distance & \# of EBs & Source \\
\hline LMC & $50 \mathrm{kpc}$ & 4634,26121 & MACHO, OGLE III \\
SMC & $60 \mathrm{kpc}$ & 1509,1350 & MACHO, OGLE II \\
NGC 6822 & $460 \mathrm{kpc}$ & 3 & Araucaria Project \\
IC 1613 & $730 \mathrm{kpc}$ & 1 & Araucaria Project \\
M31 & $750 \mathrm{kpc}$ & $\sim 500$ & DIRECT Project \& Ribas et al. $(2004)$ \\
M33 & $960 \mathrm{kpc}$ & 148 & DIRECT Project \\
\hline
\end{tabular}

Table 1 presents a census of known EBs in Local Group galaxies for which variability surveys have been conducted. The large number of systems in the Magellanic Clouds is owing to the OGLE and MACHO microlensing surveys (Faccioli et al. 2007; Graczyk et al. 2011). Moving farther out, the dwarf galaxy eclipsing systems in IC 1613 and NGC 6822 were discovered by the Araucaria project. Finally, the significant number of systems discovered in M31 and M33 originate from the dedicated searches by the DIRECT Project (e.g., Stanek et al. 1998; Ribas et al. 2004). EBs have thus far been used as distance indicators to the Large Magellanic Cloud (LMC; e.g., Guinan et al. 1998; Fitzpatrick et al. 2003; Bonanos et al. 2011), the Small Magellanic Cloud (SMC; e.g., Harries et al. 2003; Hilditch et al. 2005; North et al. 2010), M31 (Ribas et al. 2005; Vilardell et al. 2010) and M33 (Bonanos et al. 2006).

\section{Large Magellanic Cloud}

As one of the nearest galaxies to the Milky Way, the LMC has naturally been an attractive first rung for the extragalactic distance scale. The Hubble Space Telescope Key Project (Freedman et al. 2001) adopted a distance modulus $\mu=18.50 \pm 0.10$ mag (corresponding to a distance of $50.1 \pm 2.4 \mathrm{kpc}$ ) to the $\mathrm{LMC}$, which has become the consensus in the community. Schaefer (2008) pointed out that bandwagon effects are present in the literature, with pre-2001 LMC distance measurements yielding values between 18.1 and $18.8 \mathrm{mag}$ and post-2001 values clustering around the Key Project value. Given that different systematic errors accompany each method, a careful comparison of the distances resulting from different methods is necessary to characterize them. Furthermore, there is increasing evidence of substantial and complex vertical structure in the disk of the LMC (van der Marel 2006) from studies of red clump stars (Olsen \& Salyk 2002; Subramanian \& Subramanian 2010), Cepheid variables (Nikolaev et al. 2004), and RR Lyrae stars (Pejcha \& Stanek 2009), which demands further exploration.

EB distances have been measured to four early-B stars (Fitzpatrick et al. 2003), one O-star (Bonanos et al. 2011), and one G-type giant in the LMC (Pietrzyński et al. 
2009). Five of these systems are located within the bar of the LMC and their individual distances are consistent with the quoted uncertainties. A sixth system, located several degrees away in the north-eastern quadrant of the LMC's disk, gives a $3 \sigma$ shorter distance of $43.2 \pm 1.8 \mathrm{kpc}$. Fig. 1 (from Bonanos et al. 2011) shows the spatial distribution of all known EBs, and the systems with measured distances, overlaid onto the Spitzer SAGE image in the IRAC $3.6 \mu \mathrm{m}$ band (Meixner et al. 2006). A magnitude cut ( $V<17 \mathrm{mag}$ ) and period cut ( $>1.5$ days) were both applied to the EB catalogs to reject foreground systems and faint systems whose immediate follow-up is unrealistic or impossible. The detached EBs selected by Michalska \& Pigulski (2005) from among the OGLE II systems as most suitable for distance determination are also shown. Both the Hi kinematic center (Kim et al. 1998) and the dynamical center are overplotted, as is the line of nodes (van der Marel et al. 2002).

Motivated by the evidence of vertical structure in the LMC and the one discrepant EB distance, we proceeded to compute the distance to LMC-SC1-105. $†$ LMC-SC1-105 is a massive, semi-detached, short-period ( $P=4.25$ days) O-type system, with component masses of $M_{1}=30.9 \pm 1.0 \mathrm{M}_{\odot}$ and $M_{2}=13.0 \pm 0.7 \mathrm{M}_{\odot}$, and radii of $R_{1}=15.1 \pm 0.2 \mathrm{R}_{\odot}$

$\dagger$ Also known as OGLE J053448.26-694236.4 = MACHO 81.8881.21 = LH 81-72.

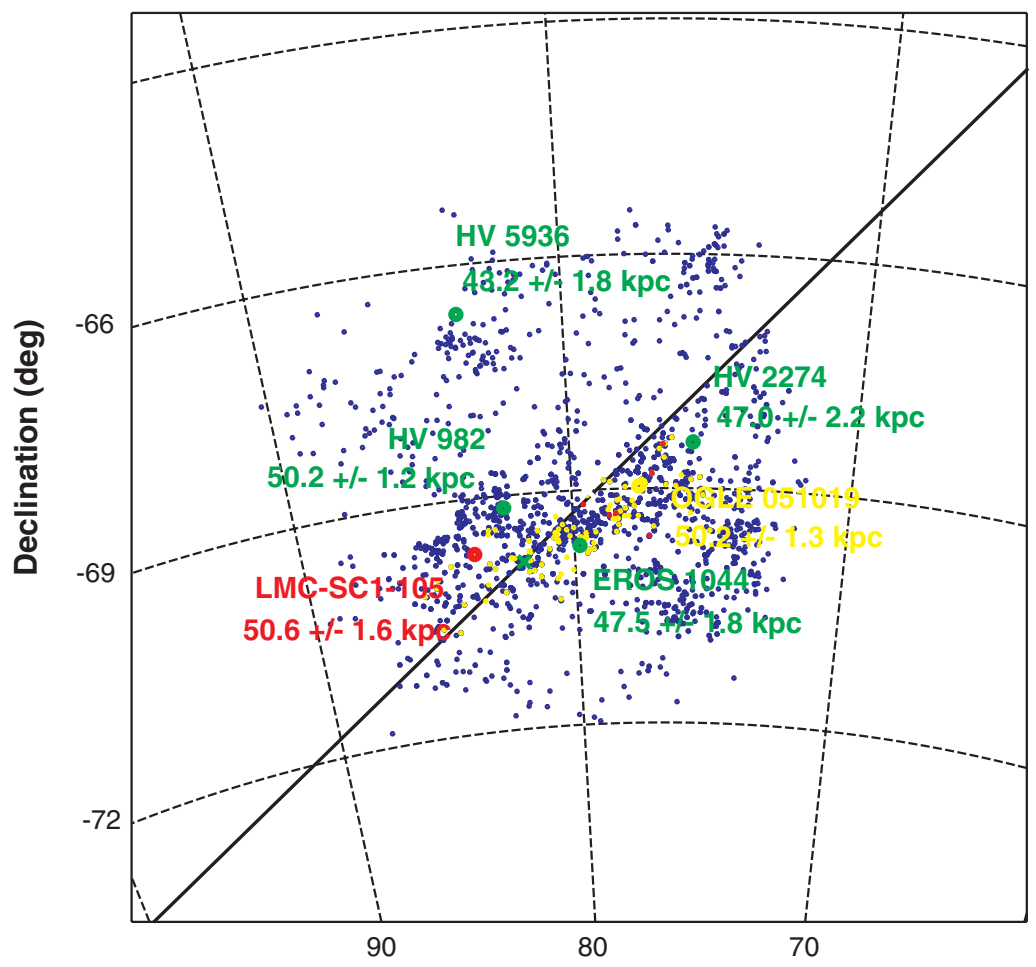

R.A. (deg)

Figure 1. Spatial distribution of known EBs on the Spitzer 3.6 $\mu \mathrm{m}$ image of the LMC (from Bonanos et al. 2011). EBs with measured distances are labelled. Yellow circles mark the most suitable detached EBs for distance determination Michalska \& Pigulski (2005); red circles mark the OGLE II binaries we plan to measure distances to next. The Hi kinematic center (white ' $X$ ') from Kim et al. (1998) and the dynamical center or center of the bar (green ' $x$ ') from van der Marel et al. (2002) are labelled; the solid line corresponds to the line of nodes (van der Marel et al. 2002). Coordinates are given for J2000. 
and $R_{2}=11.9 \pm 0.2 \mathrm{R}_{\odot}$ (Bonanos et al. 2009). In Bonanos et al. (2011), we determined the distance to LMC-SC1-105 - and consequently the LMC bar - at 50.6 $\pm 1.6 \mathrm{kpc}(\mu=$ $18.52 \pm 0.07 \mathrm{mag})$. The agreement we found with previous EB distances to systems in the bar of different spectral types testified to the robustness of the EB method and its potential as a powerful, independent distance indicator. Furthermore, it confirmed that O-type (and semi-detached) EBs are suitable for distance determination, i.e. that the fluxes predicted by FASTWIND are indeed accurate.

\section{IC 1613}

The Araucaria Project has discovered a luminous, blue EB in the low-metallicity, dwarf irregular galaxy IC 1613. We are deriving the first direct EB distance to this galaxy, with the purpose of comparing our result with that obtained from other distance indicators (e.g., Cepheids; Pietrzyński et al. 2006). Since young stars in IC 1613 have the lowest known metallicity $([\mathrm{Fe} / \mathrm{H}]=-1.0$ dex; Freedman et al. 1988; Bresolin et al. 2007), such a comparison will provide very valuable information about the dependence of various distance indicators on metallicity.

We have obtained a $V$-band light curve from the OGLE $1.3 \mathrm{~m}$ telescope, and additional data from the Aristarchos $2.3 \mathrm{~m}$ telescope, shown in Fig. 2. We find that the system is detached and has a period of 4.4 days. We have also obtained high-resolution spectra with MagE on the $6.5 \mathrm{~m}$ Magellan and UVES on the $8 \mathrm{~m}$ VLT telescopes. Careful modeling based on FASTWIND following the method outlined in Bonanos et al. (2011) and Castro et al. (2012) yielded radial velocities, which are plotted in the radial velocity curve shown in Fig. 2.
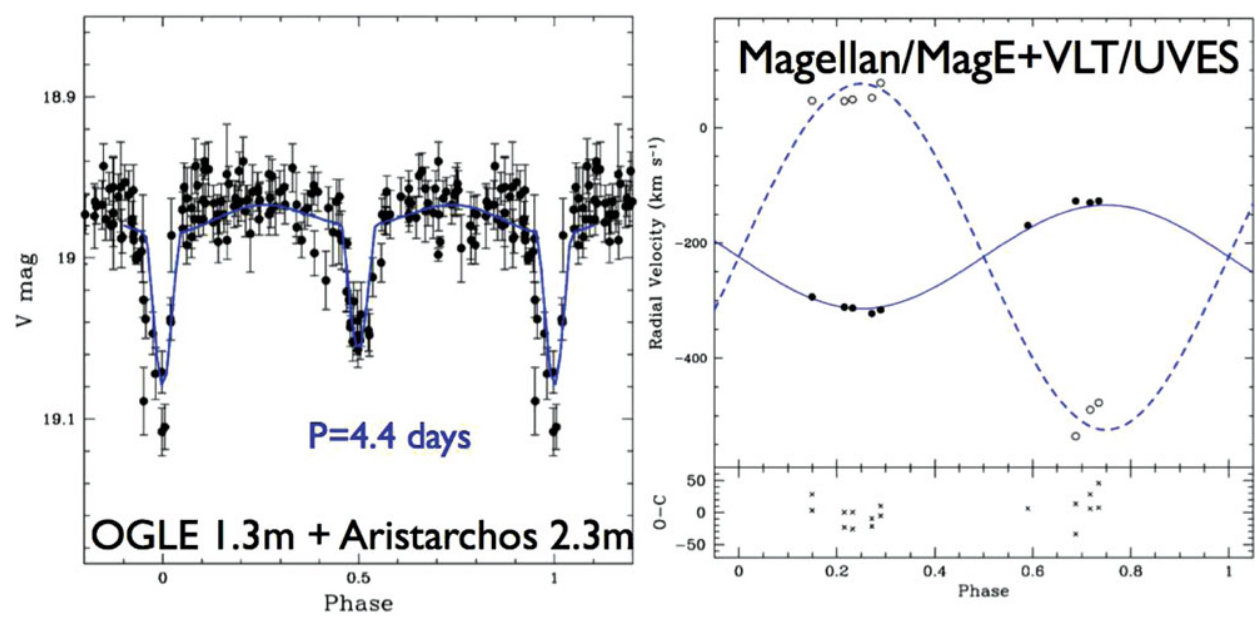

Figure 2. (left) $V$-band light curve from the OGLE $1.3 \mathrm{~m}$ and Aristarchos $2.3 \mathrm{~m}$ telescopes of the EB in IC 1613. (right) Radial velocity curves of the system measured from spectra obtained with Magellan/MagE and VLT/UVES. Blue curves indicate preliminary solutions for a detached system in a circular orbit, with a period of 4.4 days.

\section{The M33 'distance controversy'}

The proximity of M31 and M33 renders them key galaxies for the distance scale, in particular for calibrating fainter distance indicators and increasing the number of nearby 
anchor galaxies. The DIRECT Project set out to determine direct and accurate EB distances to both galaxies. It discovered 674 and 820 variables in each galaxy, respectively (e.g., Stanek et al. 1998; Bonanos et al. 2003), including a handful of detached EBs suitable for distance determination. We should stress that these are the first detached EBs ever found in M31 and M33. We obtained more precise $B V$ photometry for the two brightest systems in M33 ( $V=19-20 \mathrm{mag})$ with the KPNO $2.1 \mathrm{~m}$ telescope and followed up the brightest star with spectroscopy, employing Keck/ESI and Gemini/GMOS. We also obtained infrared photometry with NIRI on Gemini to constrain interstellar extinction. We derived a distance modulus of $24.92 \pm 0.12 \mathrm{mag}(964 \pm 54 \mathrm{kpc})$, i.e. with a $6 \%$ accuracy, which is $\sim 0.3 \mathrm{mag}$ or $13 \%$ longer than the Cepheid distance to M33 (Bonanos et al. 2006). The so-called M33 'distance controversy' remains unresolved. Scowcroft et al. (2009) obtained a Cepheid distance of $24.53 \pm 0.11 \mathrm{mag}$, while U et al. (2009) obtained distances of $24.93 \pm 0.11 \mathrm{mag}$ with the flux-weighted gravity-luminosity relation and $24.84 \pm 0.10 \mathrm{mag}$ using the tip of the red giant branch. The latter authors attribute the discrepancy to the erroneous determination of the extinction and the reddening law.

An additional EB distance to M33 would verify our result and help resolve this discrepancy. We have obtained 12 epochs of spectroscopy with Gemini/GMOS of the fainter system, D33J013337.0+303032.8 or M33B (Macri et al. 2001), an eccentric detached system with a 6.16 day period, and near-infrared photometry with Gemini/NIRI to constrain the reddening. Furthermore, we obtained $V$-band photometry with the $2.4 \mathrm{~m} M D M$ telescope in the Fall of 2011. Our preliminary analysis of the spectra yielded late-O spectral types for the components and preliminary fits to the light and radial velocity curves yielded massive components. Once all the photometry has been incorporated into the analysis, we will obtain a second accurate and direct distance determination to M33 with the aim of resolving the M33 'distance controversy.'

\section{Conclusions}

In conclusion, early-type eclipsing spectroscopic binaries are valuable, robust distance indicators with the potential of achieving $1 \%$ accuracy in distances. These systems can be used to independently calibrate the extragalactic distance scale at a range of metallicities available in the Local Group, thereby providing the calibration of the zero point for various other distance indicators. Current telescope aperture sizes limit the applicable range of the method to $1 \mathrm{Mpc}$, or to the edge of the Local Group. Pursuing EB distance measurements is therefore important for providing additional anchor galaxies for the extragalactic distance scale. It is also important for calibrating the various distance indicators as a function of metallicity. Advancing the physics of massive stars (e.g., testing model atmospheres, obtaining surface brightness calibrations) is crucial to fully characterize the accuracy of the EB method.

\section{Acknowledgements}

The author acknowledges research and travel support from the European Commission's Framework Program Seven under Marie Curie International Reintegration Grant PIRG04-GA-2008-239335.

\section{References}

Andersen, J. 1991, A\&ARv, 3, 91

Bonanos, A. Z., Stanek, K. Z., Sasselov, D. D., et al. 2003, AJ, 126, 175

Bonanos, A. Z., Stanek, K. Z., Kudritzki, R. P., et al. 2006, ApJ, 652, 313 
Bonanos, A. Z. 2009, ApJ, 691, 407

Bonanos, A. Z., Castro, N., Macri, L. M., \& Kudritzki, R. P. 2011, ApJ, 729, L9

Bresolin, F., Urbaneja, M., Gieren, E., et al. 2007, ApJ, 652, 313

Castro, N., Urbaneja, M. A., Herrero, A., et al. 2012, A\&A, 542, 79

Chavez, J. M., Macri, L. M., Pellerin, A., et al. 2012, AJ, 144, 113

Faccioli, L., Alcock, C., Cook, K., et al. 2007, AJ, 134, 1963

Fitzpatrick, E. L., Ribas, I., Guinan, E. F., et al. 2003, ApJ, 587, 685

Freedman, W. L. 1988, AJ, 96, 1248

Freedman, W. L., Madore, B. F., Gibson, B. K., et al. 2001, ApJ, 553, 47

Graczyk, D., Soszynski, I., Poleski, R., et al. 2011, Acta Astron., 61, 103

Guinan, E. F., Fitzpatrick, E. L., Dewarf, L. E., et al. 1998, ApJ, 509, L21

Harries, T. J., Hilditch, R. W., \& Howarth, I. D. 2003, MNRAS, 339, 157

Hilditch, R. W., Howarth, I. D., \& Harries, T. J. 2005, MNRAS, 357, 304

Kim, S., Staveley-Smith, L., \& Dopita, M. A. 1998, ApJ, 503, 674

Macri, L. M., Stanek, K. Z., Sasselov, D. D., et al. 2001, AJ, 121, 870

Meixner, M., Gordon, K. D., Indebetouw, R., et al. 2006, AJ, 132, 2268

Michalska, G. \& Pigulski, A. 2005, A\&A, 434, 89

Nikolaev, S., et al. 2004, ApJ, 601, 260

North, P., Gauderon, R., Barblan, F., \& Royer, F. 2010, A\&A, 520, 74

Olsen K. A. G., Salyk, C. 2002, AJ, 124, 2045

Paczyński, B. 1997, in: The Extragalactic Distance Scale, STScI Symp. Ser. (Livio, M., ed.), Cambridge Univ. Press, p. 273

Pejcha, O. \& Stanek, K. Z. 2009, ApJ, 704, 1730

Pietrzyński, G., Gieren, W., Soszynski, I., et al. 2006, AJ, 128, 2815

Pietrzyński, G., Thompson, I. B., Graczyk, D., et al. 2009, ApJ, 697, 862

Ribas, I., Jordi, C., Vilardell, F., et al. 2004, New Astron. Rev., 48, 755

Ribas, I., Jordi, C., Vilardell, F., et al. 2005, ApJ, 635, L37

Schaefer, B. E. 2008, AJ, 135, 112

Scowcroft, V., Bersier, D., Mould, J. R., \& Wood, P. R. 2009, MNRAS, 396, 1287

Stanek, K. Z., Kaluzny, J., Krockenberger, M., et al. 1998, AJ, 115, 1894

Subramanian, S. \& Subramanian, A. 2010, A\& A, 520, 24

Torres, G., Andersen, J., \& Gimenez, A. 2010, A\&ARv, 18, 67

U, V., Urbaneja, M. A., Kudritzki, R.-P., et al. 2009, ApJ, 704, 1120

van der Marel, R. P., Alves, D. R., Hardy, E., et al. 2002, AJ, 124, 2639

van der Marel, R. P. 2006, in: The Local Group as an Astrophysical Laboratory (Livio, M., \& Brown, T.M., eds), p. 47

Vilardell, F., Ribas, I., Jordi, C., et al. 2010, A\&A, 509, 70 